\title{
ARTICLE
}

Molecular Diagnostics

\section{Systemic inflammation scores correlate with survival prognosis in patients with newly diagnosed brain metastases}

\author{
Angelika M. Starzer ${ }^{1,2}$, Ariane Steindl ${ }^{1,2}$, Maximilian J. Mair $\mathbb{D}^{1,2}$, Carola Deischinger ${ }^{3}$, Anika Simonovska ${ }^{1,2}$, Georg Widhalm $^{2,4}$, \\ Brigitte Gatterbauer $^{2,4}$, Karin Dieckmann ${ }^{2,5}$, Gerwin Heller $\mathbb{D}^{1,2}$, Matthias Preusser ${ }^{1,2}$ and Anna S. Berghoff (DD ${ }^{1,2}$
}

BACKGROUND: Systemic inflammation measured by the neutrophil-to-lymphocyte ratio (NLR), leucocyte-to-lymphocyte ratio (LLR), platelet-to-lymphocyte ratio (PLR), monocyte-to-lymphocyte ratio (MLR) and CRP/albumin ratio (CRP/Alb) was shown to impact the survival prognosis in patients with extracranial solid cancer.

METHODS: One thousand two hundred and fifty patients with newly diagnosed brain metastases (BM) were identified from the Vienna Brain Metastasis Registry.

RESULTS: PLR and CRP/Alb were higher in patients with progressive extracranial disease and lower in patients with no evidence of extracranial disease. Lower NLR (cut-off $=5.07 ; 9.3$ vs. 5.0 months), LLR (cut-off $=5.76 ; 10.0$ vs. 5.3 months), PLR (cut-off $=335$; 8.0 vs. 3.8 months), MLR (cut-off $=0.53 ; 6.0$ vs. 3.5 months) and CRP/Alb (cut-off $=2.93 ; 8.5$ vs. 3.7 months; $p_{\text {adj }}<0.05$ ) were associated with longer overall survival (OS). In multivariate analysis with graded prognostic assessment (hazard ratio (HR) 1.45; 95\% confidence interval (Cl): 1.32-1.59; $p_{\text {adj }}=1.62 \mathrm{e}-13$, NLR (HR 1.55; 95\% Cl: $\left.1.38-1.75 ; p_{\text {adj }}=1.92 \mathrm{e}-11\right)$, LLR (HR 1.57; 95\% Cl: $1.39-1.77 ; p_{\text {adj }}=1.96 \mathrm{e}-11$ ), PLR (HR 1.60; 95\% Cl: $\left.1.39-1.85 ; p_{\text {adj }}=2.87955 \mathrm{e}-9\right)$, MLR (HR $1.41 ; 95 \%$ Cl: $\left.1.14-1.75 ; p_{\text {adj }}=0.027\right)$ and CRP/Alb (HR 1.83; 95\% Cl: 1.54-2.18; $p_{\text {adj }}=2.73 \mathrm{e}-10$ ) remained independent factors associated with OS at BM diagnosis. CONCLUSIONS: Systemic inflammation, measured by NLR, LLR, PLR, MLR and CRP/Alb, was associated with OS in patients with BM. Further exploration of immune modulating therapies is warranted in the setting of BM.

British Journal of Cancer (2021) 124:1294-1300; https://doi.org/10.1038/s41416-020-01254-0

\section{BACKGROUND}

Brain metastases (BMs) are a frequent and life limiting complication in solid cancers. ${ }^{1}$ Immune checkpoint inhibitor-based therapies have shown activity in patients with BM. ${ }^{1-4}$ However, the clinical efficacy is higher in patients with asymptomatic BM compared to patients suffering from neurological symptoms in need for steroid treatment. ${ }^{3}$ Indeed, immune responses are tightly controlled in the brain in order to avoid any harmful damage to this sensitive organ. ${ }^{5}$ Previously, the density of intratumoural $\mathrm{T}$ cells was described as a favourable prognostic factor in BM patients underscoring the prognostic importance of cancer-immune system interactions also in patients with $\mathrm{BM}^{6}{ }^{6}$ Recent insights into the cancer-immune system interactions further stressed the importance of systemic inflammation in addition to the local inflammatory characteristics. ${ }^{7}$ Systemic inflammatory scores, including neutrophil-to-lymphocyte ratio (NLR), leucocyte-to-lymphocyte ratio (LLR), platelet-tolymphocyte ratio (PLR), monocyte-to-lymphocyte ratio (MLR) and C-reactive protein/albumin ratio (CRP/Alb), were shown to impact tumour control and in consequence survival prognosis of patients with extracranial metastatic cancer. ${ }^{8-12}$ Moreover, markers of systemic inflammation, including pre-treatment CRP, NLR and $M L R$, were recently shown to be associated with the progressionfree survival (PFS) and overall survival (OS) in patients treated with immune checkpoint inhibitor-based therapy. ${ }^{13-15}$ However, the prognostic impact of systemic inflammation was not yet investigated in a comprehensive real-life cohort of BM patients. Therefore, we aimed to analyse systemic inflammation measured by the NLR, LLR, PLR, MLR and CRP/Alb and their correlation with survival prognosis in a large real-life cohort of patients with BM.

\section{METHODS}

Patients and data collection

Patients with newly diagnosed BM from solid tumours and treated at the Medical University of Vienna between 1990 and 2019 were identified from the Vienna Brain Metastasis Registry. Investigated markers of systemic inflammation included the NLR, LLR, PLR, MLR and CRP/Alb at the time of BM diagnosis \pm 14 days. Steroid therapy at $\mathrm{BM}$ diagnosis was defined as \pm 14 days from diagnosis of $\mathrm{BM}$. Data closest to the diagnosis of BM was chosen for analysis. Synchronous diagnosis of BM with primary cancer or progressive extracranial disease (new or growing extracranial lesions) is defined as \pm 30 days from BM diagnosis. The graded prognostic assessment (GPA) score of our patient cohort was calculated based on clinical parameters, which include age, Karnofsky performance score, number of $\mathrm{BM}$ and the status of the

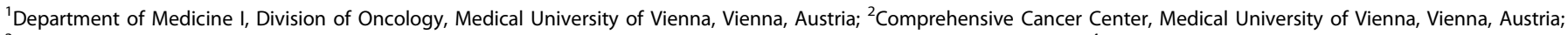

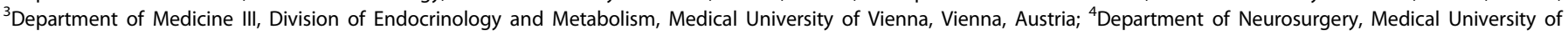
Vienna, Vienna, Austria and ${ }^{5}$ Department of Radiotherapy, Medical University of Vienna, Vienna, Austria

Correspondence: Anna S. Berghoff (anna.berghoff@meduniwien.ac.at)

Received: 6 July 2020 Revised: 7 December 2020 Accepted: 17 December 2020

Published online: 21 January 2021 
extracranial disease, as previously described. ${ }^{16}$ The ACCI was calculated as previously published. ${ }^{17}$ For each included comorbidity a score was given. A total score was calculated by the sum of scores for each comorbidity and for each decade starting with 50 years 1 point was added (e.g. addition of 1 point for age group 50-59 years). ${ }^{11}$ The studied patient cohort was treated independently by multidisciplinary teams according to good clinical practice guidelines. This project was approved by the ethics committee of the Medical University of Vienna (078/2004).

\section{Statistical analysis}

Cancer entities occurring $<10$ times in the entire cohort were summarised under the primary cancer entity "others". The Kolmogornov-Smirnov test was used to test for data normality. Differences between groups were assessed using the Kruskal-Wallis test. Correlations of metric variables were determinated using the Spearman's rho, while a correlation coefficient of $\rho>0.7$ was interpreted as strong correlation, $0.7 \geq \rho>0.5$ as medium correlation, $0.5 \geq \rho>0.3$ as weak correlation and $<0.3$ as no correlation. OS from diagnosis of $\mathrm{BM}$ was defined as time from radiological diagnosis of BM until death or last follow-up. Patient stratification cut-offs for survival analyses were calculated according to the maximally selected rank statistics using the $\mathrm{R}$ package maxstat that iteratively tests all possible cut-points to identify the value with the maximum rank statistics for optimal group stratification for survival analyses. $^{18,19}$ The Kaplan-Meier product limit method was used to illustrate survival times and log-rank tests were calculated to estimate survival differences between groups. Survival analyses were calculated using the $\mathrm{R}$ packages survival and survminer. ${ }^{20,21}$ A multivariate analysis using the Cox proportional hazard model was applied to adjust for the GPA score as an established prognostic assessment. ${ }^{16} P$ values were Bonferroni adjusted for 27 applied statistical tests resulting in adjusted $p$ values $\left(p_{\text {adj }}\right)$ for each statistical test. A two-tailed $p_{\mathrm{adj}}<0.05$ was considered to indicate statistical significance. Statistical analyses were performed using the Statistical Package for the Social Sciences (SPSS $\left.{ }^{\circledR}\right) 23.0$ software (SPSS Inc., Chicago, IL, USA) and R (R Foundation for Statistical Computing, Vienna, Austria).

\section{RESULTS}

Patients characteristics

One thousand two hundred and fifty patients (662/1250, 53\% males; 588/1250, 47\% females) with a median age of 62 years (range 23-91) at diagnosis of BM were included in the analysis. Five hundred and seventy-one of 1250 (45.7\%) patients were diagnosed with BM simultaneously with diagnosis of the primary tumour. One hundred and eight of 1250 (8.6\%) patients showed no evidence of extracranial disease at BM diagnosis, while stable extracranial disease at BM diagnosis was evident in $233 / 1250$ (18.6\%) patients. Three hundred and thirty-eight of 1250 (27.0\%) patients presented with synchronous progressive extracranial disease. One thousand one hundred and sixty-nine of 1250 $(93.5 \%)$ patients were locally treated with neurosurgical resection (7\%), radiotherapy $(77.8 \%)$ or a combination of surgery and radiotherapy (8.7\%) of $\mathrm{BM}$, while $34 / 1250$ (2.7\%) of patients received systemic therapy or best supportive care only $(47 / 1250$, $3.8 \%)$. Patients receiving systemic therapy as first-line treatment for BM showed the longest OS from diagnosis of BM (15 months), followed by a combinational therapy of surgery plus radiotherapy (14 months), surgery only (7 months), radiotherapy only ( 6 months) and best supportive care (1 month; log-rank test; $\left.p_{\text {adj }}=1.6 \mathrm{e}-20\right)$. Median survival in the entire cohort was 6 months (range 0-178 months; Table 1). GPA class showed a significant association with survival prognosis from diagnosis of BM in univariate analysis (hazard ratio (HR) 1.47; 95\% confidence interval (Cl): $1.35-1.62 ; p_{\text {adj }}=2.10 \mathrm{e}-15$; Cox regression model); Supplementary Fig. 1).
Table 1. Patients characteristics.

\begin{tabular}{|c|c|c|}
\hline Characteristics & $n=1250$ & $100 \%$ \\
\hline \multicolumn{3}{|l|}{ Age at diagnosis of $\mathrm{BM}$, years } \\
\hline Median (range) & $62(23-91)$ & \\
\hline \multicolumn{3}{|l|}{ Sex } \\
\hline Male & 662 & 53 \\
\hline Female & 588 & 47 \\
\hline \multicolumn{3}{|l|}{ Cancer entity } \\
\hline Lung cancer & 994 & 79.5 \\
\hline Breast cancer & 86 & 6.9 \\
\hline Melanoma & 106 & 8.5 \\
\hline Renal cell carcinoma & 7 & 0.6 \\
\hline Colorectal cancer & 11 & 0.9 \\
\hline CUP & 10 & 0.8 \\
\hline Others & 36 & 2.9 \\
\hline \multicolumn{3}{|l|}{ Surgery of the primary tumour } \\
\hline Yes & 359 & 28.7 \\
\hline No & 891 & 71.3 \\
\hline \multicolumn{3}{|l|}{ Radiotherapy to the primary tumour site } \\
\hline Yes & 221 & 17.7 \\
\hline No & 1029 & 82.3 \\
\hline \multicolumn{3}{|l|}{ Adjuvant chemotherapy } \\
\hline Yes & 346 & 27.7 \\
\hline No & 904 & 72.3 \\
\hline \multicolumn{3}{|l|}{ KPS } \\
\hline Median (range) & $80(0-100)$ & \\
\hline \multicolumn{3}{|l|}{ GPA class } \\
\hline Class I & 41 & 3.3 \\
\hline Class II & 121 & 9.7 \\
\hline Class III & 761 & 60.9 \\
\hline Class IV & 327 & 26.2 \\
\hline \multicolumn{3}{|l|}{ Status of extracranial disease } \\
\hline $\begin{array}{l}\text { Synchronous diagnosis of BM at cancer } \\
\text { diagnosis }\end{array}$ & 571 & 45.7 \\
\hline No evidence of extracranial disease & 108 & 8.6 \\
\hline Stable disease & 233 & 18.6 \\
\hline Progressive disease & 338 & 27.0 \\
\hline \multicolumn{3}{|l|}{ Chemotherapy before diagnosis of BM } \\
\hline Yes & 581 & 46.5 \\
\hline No & 669 & 53.5 \\
\hline \multicolumn{3}{|l|}{ Steroid treatment at BM diagnosis } \\
\hline Yes & 479 & 38.3 \\
\hline No & 728 & 58.2 \\
\hline NA & 43 & 3.4 \\
\hline \multicolumn{3}{|l|}{ First-line treatment of BM } \\
\hline Surgery & 87 & 7.0 \\
\hline Radiotherapy total & 973 & 77.8 \\
\hline (GK/WBRT/GK + WBRT) & $(597 / 280 / 96)$ & $\begin{array}{l}(47.8 / 22.4 / \\
7.6)\end{array}$ \\
\hline $\begin{array}{l}\text { (Radiotherapy within } 14 \text { days of BM } \\
\text { diagnosis, } n=973^{*} \text { ) }\end{array}$ & (432) & $\left(44.4^{*}\right)$ \\
\hline $\begin{array}{l}\text { Combinational local therapy (surgery }+ \\
\text { radiotherapy) }\end{array}$ & 109 & 8.7 \\
\hline Systemic therapy & 34 & 2.7 \\
\hline BSC & 47 & 3.8 \\
\hline \multicolumn{3}{|l|}{ Overall survival, months } \\
\hline Median (range) & $6(0-178)$ & \\
\hline Alive & 151 & 12.1 \\
\hline Deceased & 1099 & 87.9 \\
\hline
\end{tabular}

$B M$ brain metastasis, CUP cancer of unknown primary, KPS Karnofsky performance status, GPA graded prognostic assessment, NA not available, GK gamma knife, WBRT whole-brain radiotherapy, BSC best supportive care. *Total number of patients having had radiotherapy. 
Table 2. Systemic inflammation scores.

\begin{tabular}{lcl}
\hline Characteristics & Median & Range \\
\hline Leucocytes G/L, $n=1250$ & 8.69 & $0.89-48.8$ \\
Neutrophils G/L, $n=1250$ & 6.15 & $0.24-66.0$ \\
Lymphocytes G/L, $n=1250$ & 1.3 & $0.1-26.5$ \\
Platelets G/L, $n=1250$ & 272.0 & $2.0-894.0$ \\
Monocytes G/L, $n=379$ & 0.7 & $0.01-2.42$ \\
CRP, $n=1190$ & 1.22 & $0-43.7$ \\
Albumin, $n=612$ & 39.8 & $15.7-53.38$ \\
NLR, $n=1250$ & 4.76 & $0.07-98.0$ \\
LLR, $n=1250$ & 6.39 & $0.12-997.5$ \\
PLR, $n=1250$ & 207.57 & $3.08-1675.0$ \\
MLR, $n=379$ & 0.52 & $0.01-3.06$ \\
CRP/Alb, $n=601$ & 2.41 & $0.02-121.64$ \\
\hline CRP C-r. & &
\end{tabular}

$C R P$ C-reactive protein, $N L R$ neutrophil-to-lymphocyte ratio, $L L R$ leucocyteto-lymphocyte ratio, $P L R$ platelet-to-lymphocyte ratio, $M L R$ monocyte-tolymphocyte ratio, CRP/Alb C-reactive protein/albumin ratio.

Systemic inflammation in patients with newly diagnosed brain metastases

NLR, LLR and PLR were available in all included patients, while MLR was available in 379/1250 (30.3\%) and CRP/Alb in 601/1250 $(48.1 \%)$ patients (Table 2). No differences in NLR, LLR, PLR, MLR or CRP/Alb according to primary tumour type was observed ( $p_{\text {adj }}>$ 0.05; Kruskal-Wallis test; Supplementary Fig. 2).

There were no correlations of systemic inflammation scores with age nor with KPS nor with age-adjusted Charlson-comorbidity index at BM diagnosis (Spearman correlation coefficient $<0.3$ ).

Twenty-one of $1250(1.7 \%)$ patients in this cohort showed a history of autoimmune disease. There was no correlation of investigated inflammation markers at BM diagnosis and history of autoimmune disease (Mann-Whitney $U$ test, $p_{\text {adj }}>0.05$ ).

PLR and CRP/Alb showed significant differences according to the status of the extracranial disease. PLR was highest in patients with progressive extracranial disease (median $\mathrm{PLR}=225.0$ ), followed by patients with stable disease (median PLR $=220.0$ ) and patients with synchronous diagnosis of BM with the primary cancer (median PLR $=196.1$ ) and lowest in patients with no evidence of extracranial disease at BM diagnosis (median $=$ PLR 189.1; $p_{\text {adj }}=0.002$; Kruskal-Wallis test; Fig. 1a). CRP/Alb was highest in patients with progressive extracranial disease (median $\mathrm{CRP} / \mathrm{Alb}=2.85$ ), followed by patients with synchronous diagnosis of $\mathrm{BM}$ with the primary cancer (median $\mathrm{CRP} / \mathrm{Alb}=2.73$ ), followed by patients with stable disease (median $\mathrm{CRP} / \mathrm{Alb}=2.06$ ) and lowest in patients with no evidence of extracranial disease (median CRP/Alb = 1.07; $p_{\text {adj }}=0.009$; Kruskal-Wallis test; Fig. 1b). NLR, LLR and MLR did not significantly differ depending on the status of the extracranial disease ( $p_{\text {adj }}>0.05$; Kruskal-Wallis test).

Patients with prior application of chemotherapy presented with a higher median PLR (median PLR 220.0) compared to chemotherapy-naive patients (median PLR 198.52; $p_{\text {adj }}=0.004$; Mann-Whitney $U$ test; Fig. 2). No differences in patients with or without prior chemotherapy were observed concerning the NLR, LLR, MLR and CRP/Alb ( $p>0.05$; Mann-Whitney $U$ test).

Four hundred and seventy-nine of 1250 (38.3\%) BM patients received steroid treatment at $\mathrm{BM}$ diagnosis. No significant differences in investigated inflammation scores were observed in patients treated with steroids compared to patients without steroid therapy ( $p_{\text {adj }}>0.05$; Mann-Whitney $U$ test).

Twenty-two of 1250 patients (1.8\%) in this cohort were treated with immune checkpoint inhibitors before the diagnosis of BM. No significant difference of inflammation markers at BM diagnosis according to therapy with immune checkpoint inhibitors prior to diagnosis of BM was observed ( $p_{\text {adj }}>0.05$; Mann-Whitney $U$ test).

Correlation of systemic inflammation markers with survival prognosis

Lower NLR (cut-off $=5.07$ ) was associated with a significantly longer OS with 9.3 months compared to 5.0 months in patients with a higher NLR ( $p_{\text {adj }}=4.98 \mathrm{e}-14$; log-rank test; Fig. 3a). Further, patients with lower LLR (cut-off $=5.76 ; 10.0$ vs. 5.3 months, $p_{\text {adj }}=$ $2.25 \mathrm{e}-14$; log-rank test; Fig. 3b), lower PLR (cut-off $=335 ; 8.0$ vs. 3.8 months; $p_{\text {adj }}=2.69 \mathrm{e}-11$; log-rank test; Fig. $3 c$ ), lower MLR (cutoff $=0.53 ; 6.0$ vs. 3.5 months; $p_{\text {adj }}=0.009$; log-rank test; Fig. $3 \mathrm{~d}$ ) and lower CRP/Alb (cut-off $=2.93 ; 8.5$ vs. 3.7 months; $p_{\text {adj }}=1.13 \mathrm{e}-12$; log-rank test; Fig. 3e) presented with a more favourable survival prognosis.

In a multivariate model adjusting for GPA (in 1250/1250 patients), NLR (HR 1.55; 95\% Cl: $1.38-1.75 ; \quad p_{\text {adj }}=1.92 \mathrm{e}-11$; Cox regression model), LLR (HR 1.57; 95\% Cl: 1.1.39-1.1.77; $p_{\text {adj }}=$ $1.96 \mathrm{e}-11$; Cox regression model), PLR (HR 1.60; 95\% Cl: 1.39-1.85; $p_{\text {adj }}=2.88 \mathrm{e}-9$; Cox regression model), MLR (HR 1.41; 95\% Cl: 1.14-1.75; $\quad p_{\text {adj }}=0.027 ;$ Cox regression model) and CRP/Alb (HR 1.83; 95\% Cl: 1.54-2.18; $p_{\text {adj }}=2.73 \mathrm{e}-10$; Cox regression model) remained independent factors associated with OS after diagnosis of BM (Table 3). In adjusting for the DS-GPA (in 1239/ 1250 patients with the DS-GPA available), the NLR (HR 1.45; 95\% Cl: $1.28-1.64 ; p_{\text {adj }}=1.30 \mathrm{e}-7$; Cox regression model), LLR (HR 1.43; 95\% Cl: 1.26-1.63; $p_{\text {adj }}=5.46 \mathrm{e}-7$; Cox regression model), PLR (HR 1.54; 95\% Cl: 1.33-1.79; $p_{\text {adj }}=2.32 \mathrm{e}-7$; Cox regression model), MLR (HR 1.40; 95\% Cl: 1.13-1.74; $p_{\mathrm{adj}}=0.05$; Cox regression model) and CRP/Alb (HR 1.71; 95\% Cl: 1.42-2.05; $p_{\text {adj }}=2.45 \mathrm{e}-7$; Cox regression model) remained independent factors associated with OS after diagnosis of BM.

\section{DISCUSSION}

Systemic inflammation scores correlated with survival prognosis in our cohort of advanced cancer patients with BM. The routinely and easily accessible NLR, LLR, PLR, MLR and CRP/Alb had independent prognostic impact in addition to the established GPA, suggesting that also in the advanced event of BM, flourishing systemic inflammatory processes are negatively associated with the course of cancer disease.

PLR and CRP/Alb were significantly higher in patients with simultaneous progressive extracranial disease at BM diagnosis compared to patients with a stable extracranial disease and patients with synchronous diagnosis of BM. In contrast, NLR, LLR and MLR did not correlate with the status of the extracranial disease. In consequence, PLR and CRP/Alb might be more determined by the status of the systemic disease than NLR, LLR and MLR. The acute-phase protein CRP increases during systemic inflammatory processes while the albumin production is reduced as an amino acid sparing mechanism. ${ }^{22}$ The Glasgow Prognostic Score includes the CRP/Alb as a prognostic marker for survival independent of cancer entity or disease stage. ${ }^{23}$ Previously, activated platelets were shown to stimulate inflammatory processes by the release of vascular endothelial growth factor and platelet-derived growth factor, which mediate the extravasation and migration of leucocytes. ${ }^{24}$ Further, platelets are postulated to contribute to cancer dissemination by depleting natural killer cells and impairing their cytotoxic activity. ${ }^{25}$ The cellbased scores NLR, LLR and MLR might reflect a more immediate impact of inflammation as neutrophils are the first effector immune cells recruited in case of acute inflammation followed by monocytes. ${ }^{26}$ Indeed, normalisation of NLR after one cycle of chemotherapy was described to result in improved PFS in colorectal cancer and mesothelioma patients. ${ }^{27,28}$ Previous chemotherapeutic treatments potentially impact the investigated inflammatory signatures. Indeed, PLR was higher in patients with 
a

$p_{\text {adj }}=0.002$

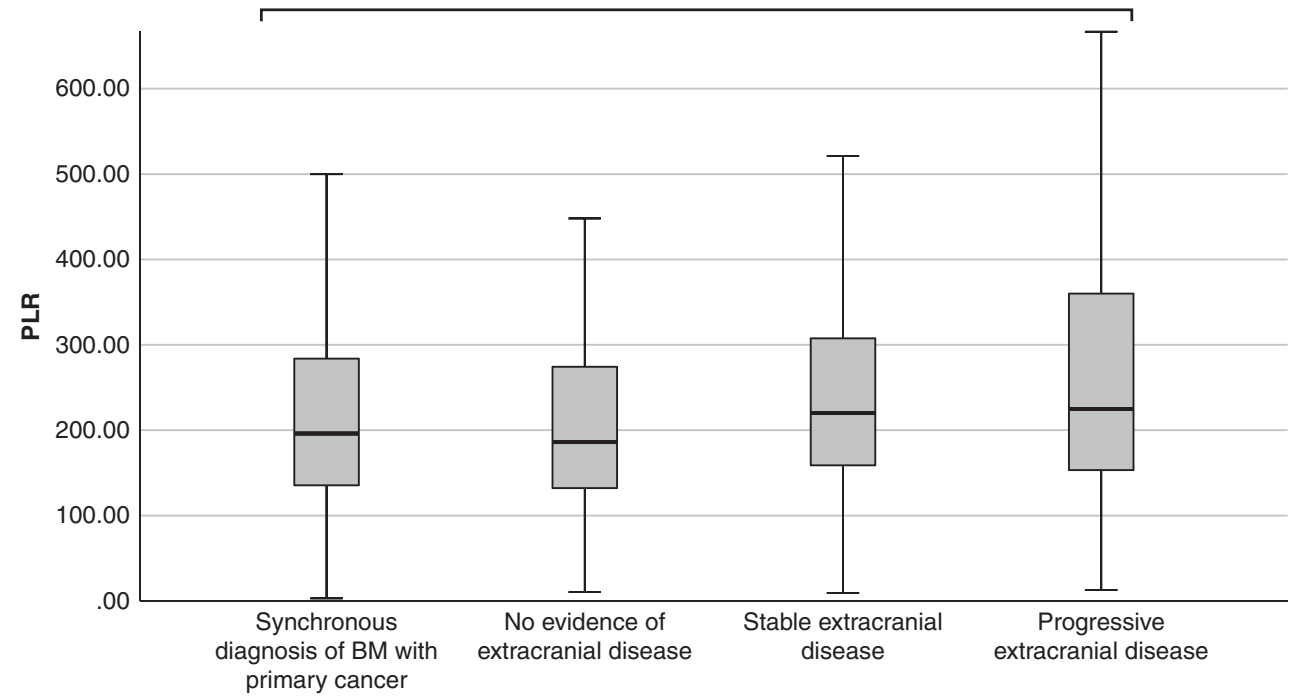

Status of extracranial disease

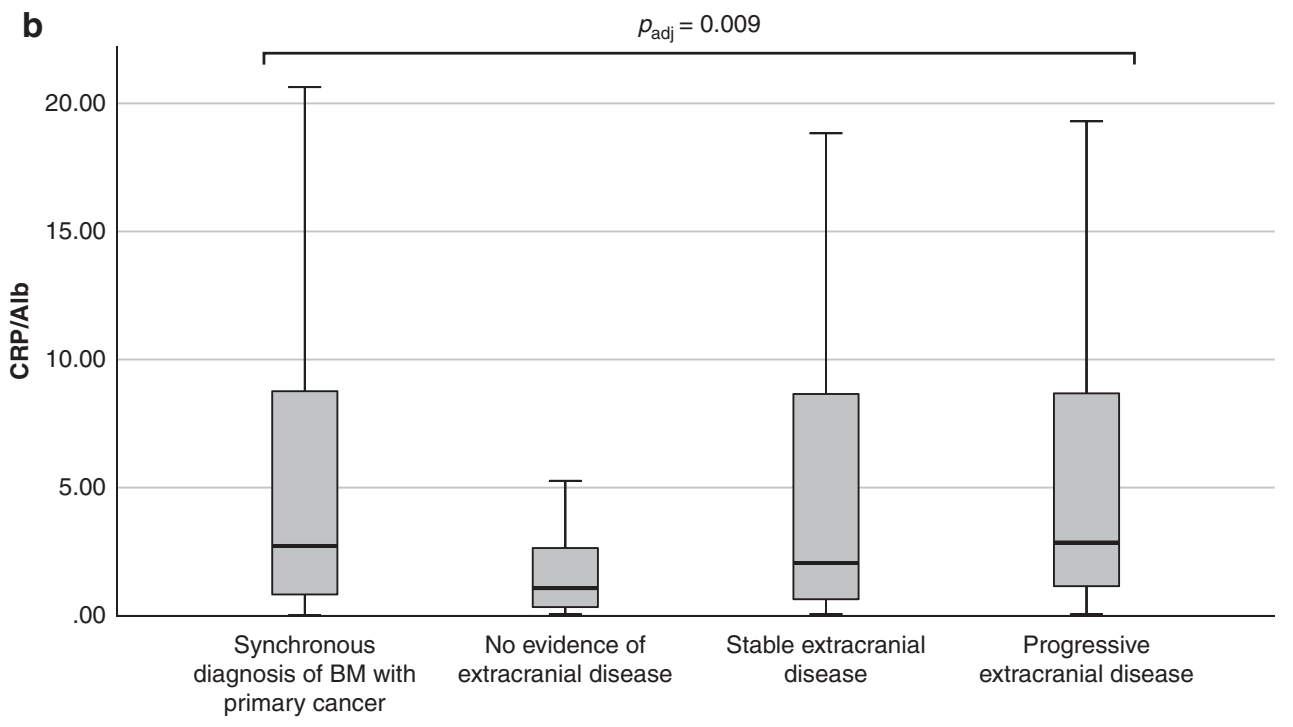

Status of extracranial disease

Fig. 1 Levels of systemic inflammation scores according to the status of the extracranial disease. a Median platelet-to-lymphocyte ratio (PLR) and $\mathbf{b}$ median C-reactive protein/albumin ratio (CRP/Alb) according to the status of the extracranial disease are highest in patients with progressive disease $(p<0.001$; Kruskal-Wallis test). Bar graphs + confidence intervals (Cls) are shown.

previous treatment, but NLR, LLR, MLR and CRP/Alb were not impacted by previous treatments. Therefore, the investigated systemic inflammatory scores give a prognostic relevant insight into the systemic inflammatory status also in pretreated BM patients.

The investigated systemic inflammation scores NLR, LLR, PLR, MLR and CRP/Alb presented with sustained prognostic impact independent from the GPA. The variable set included in the GPA does only include clinical variables like age, number of BM, Karnofsky performance score and status of the extracranial disease. ${ }^{16}$ Previously, we reported that the addition of laboratory parameters, included in the LabBM score, provide a more precise prognostic prediction than the GPA alone. ${ }^{29}$ A precise survival prediction is of particular importance in BM patients as treatment decisions have to be taken in the careful balance between efficacy and short/longterm side effects in a palliative setting. ${ }^{30}$ The CRP value was the only inflammatory marker included in the LabBM score. However, systemic inflammation is of growing importance in extracranial malignancies as, besides the prognostic impact, pre-treatment NLR, MLR, PLR and CRP were recently shown to be associated with PFS and $O S$ in melanoma, non-small cell lung cancer, renal cell carcinoma, breast and head and neck cancer patients treated with immune checkpoint inhibitor therapy. ${ }^{13-15,31,32}$ Immune checkpoint inhibitors have increasing clinical importance in BM patients as first clinical trials strongly support the application in selected patient populations with asymptomatic newly diagnosed $\mathrm{BM}^{33,34}$ None of the patients in the present series were treated with immune checkpoint inhibitors after diagnosis of BM and, therefore, future trials should investigate whether systemic inflammatory scores could have predictive potential for the response to immune checkpoint inhibitors in the BM population.

Although we investigated a particularly large, real-life cohort, the retrospective study argues for careful interpretation of the obtained data. The comprehensive set of clinical data allowed us to statistically investigate the impact of the primary tumour type, the status of the extracranial disease as well as the previous 


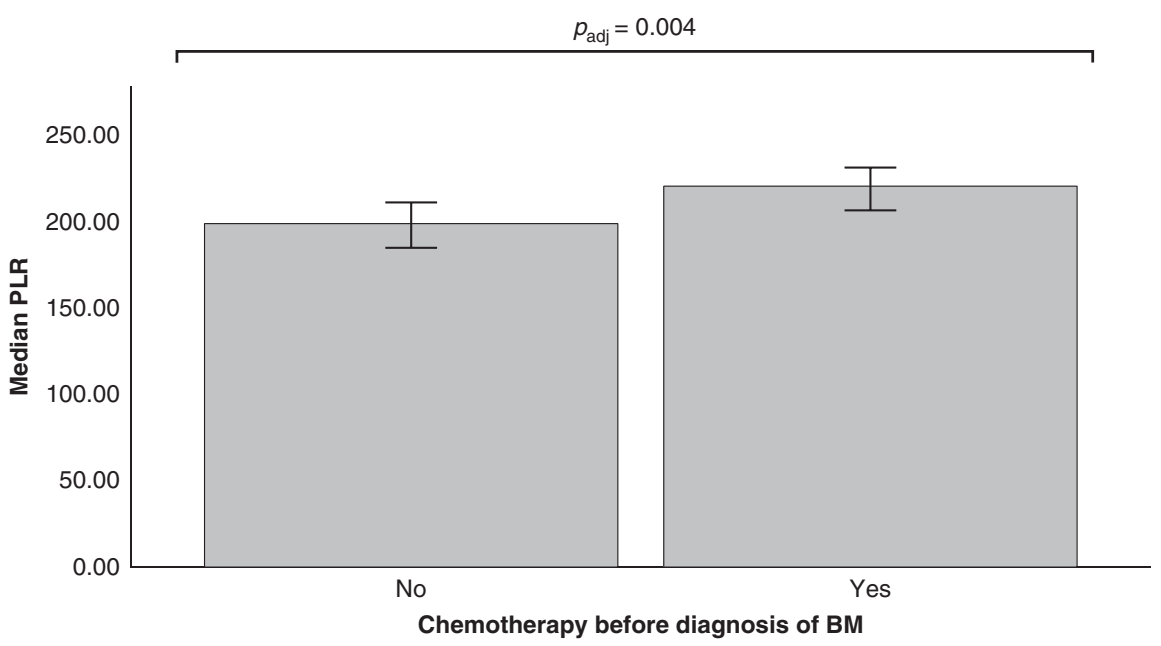

Fig. 2 Levels of platelet-to-lymphocyte ratio according to prior chemotherapy before diagnosis of BM. Median platelet-to-lymphocyte ratio (PLR) according to prior application of chemotherapy before diagnosis of BM (median PLR 220.0) compared to patients without prior chemotherapy (median PLR 198.33; $p=0.004$; Mann-Whitney $U$ test). Bar graphs + Cls are shown.

a
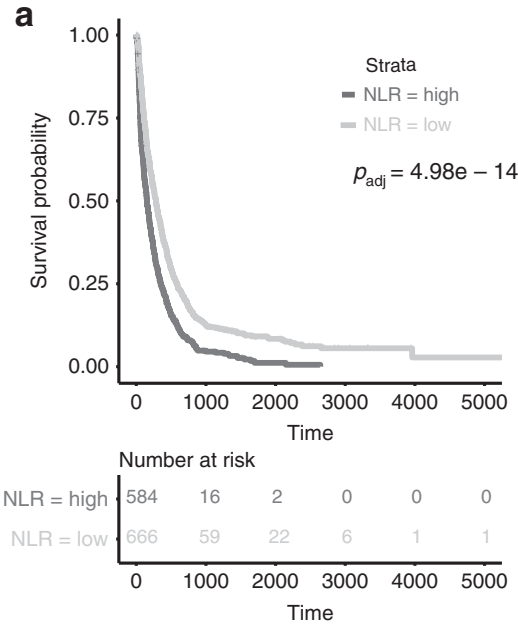

d
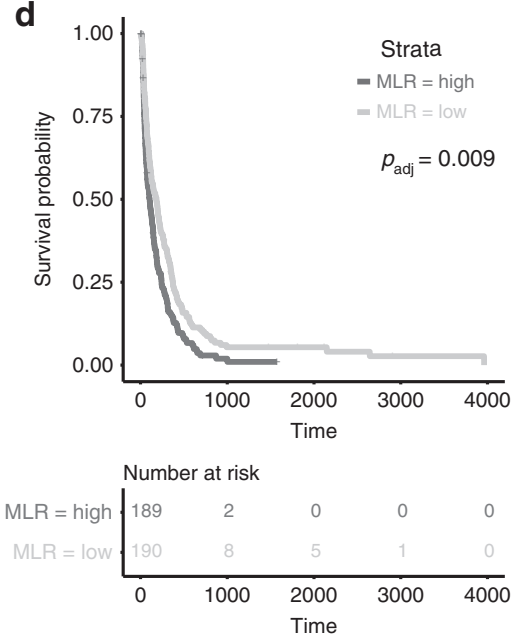

b
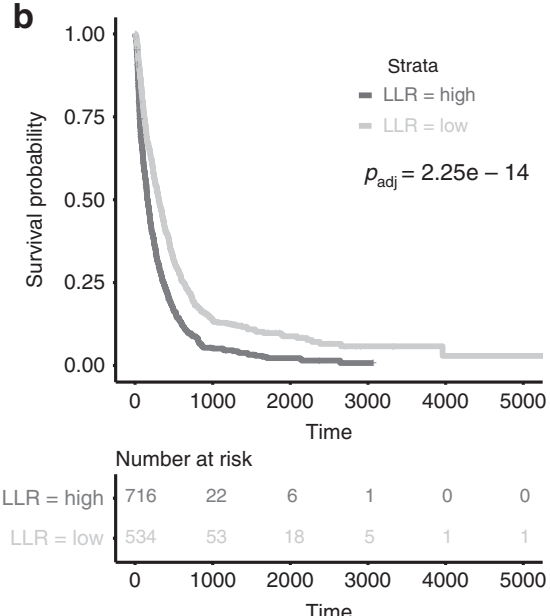

e
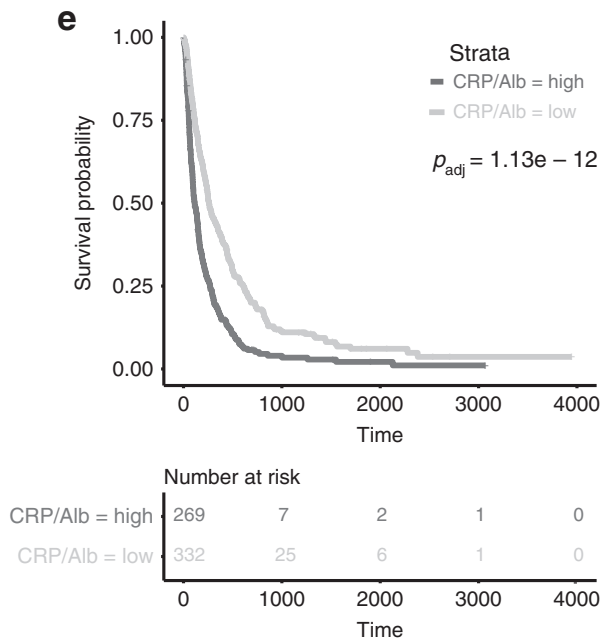
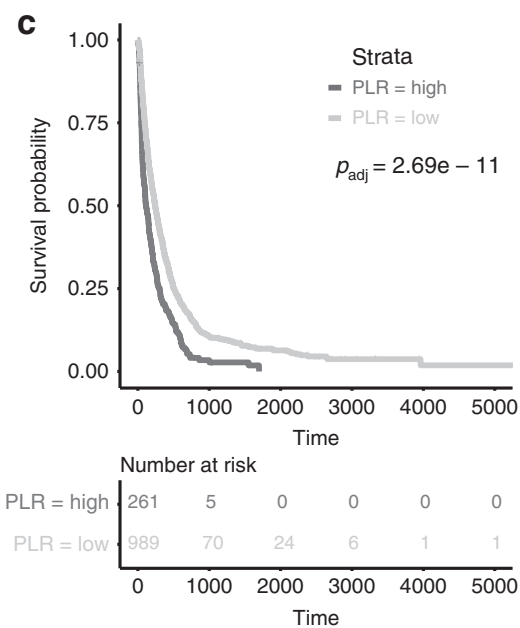

Fig. 3 Overall survival from diagnosis of BM according to markers of systemic inflammation. a Neutrophil-to-lymphocyte ratio (NLR) $\left(p_{\text {adj }}=4.98 \mathrm{e}-14\right.$; log-rank test), b Leucocyte-to-lymphocyte ratio (LLR) ( $p_{\text {adj }}=2.25 \mathrm{e}-14$; log-rank test), c Platelet-to-lymphocyte ratio (PLR) $\left(p_{\text {adj }}=2.69 \mathrm{e}-11\right.$; log-rank test), $\mathbf{d}$ Monocyte-to-lymphocyte ratio $(\mathrm{MLR})\left(p_{\text {adj }}=0.009\right.$; log-rank test), e C-reactive protein/albumin ratio (CRP/ Alb) $\left(p_{\text {adj }}=1.13 \mathrm{e}-12\right.$; log-rank test). 
Table 3. Multivariate analysis.

\begin{tabular}{llll}
\hline & \multicolumn{2}{l}{ Overall survival } & \multicolumn{1}{l}{ Cox regression } \\
\cline { 2 - 3 } & $\mathrm{HR}$ & $95 \% \mathrm{Cl}$ & \\
\hline GPA class & 1.46 & $1.32-1.59$ & $1.62 \mathrm{e}-13$ \\
NLR & 1.55 & $1.38-1.75$ & $1.92 \mathrm{e}-11$ \\
LLR & 1.57 & $1.39-1.77$ & $1.96 \mathrm{e}-11$ \\
PLR & 1.60 & $1.39-1.85$ & $2.88 \mathrm{e}-9$ \\
MLR & 1.41 & $1.14-1.75$ & 0.027 \\
CRP/Alb & 1.83 & $1.54-2.18$ & $2.73 \mathrm{e}-10$ \\
\hline
\end{tabular}

$H R$ hazard ratio, $\mathrm{Cl}$ confidence interval, GPA graded prognostic assessment, $N L R$ neutrophil-to-lymphocyte ratio, $L L R$ leucocyte-to-lymphocyte ratio, $P L R$ platelet-to-lymphocyte ratio, MLR monocyte-to-lymphocyte ratio, CRP/Alb C-reactive protein/albumin ratio.

treatments on the investigated systemic inflammatory scores. The obtained data further reflects everyday practice as values \pm 14 days from diagnosis of BM were included and certain fluctuation in the blood values could occur in this time frame. Nevertheless, the independent association in addition to GPA supports the prognostic impact. Prospective studies would be warranted to validate the observed prognostic impact of systemic inflammation in newly diagnosed BM patients.

\section{CONCLUSIONS}

In conclusion, we show that systemic inflammation scores correlate with survival prognosis in a large real-life cohort of patients with advanced cancer and brain metastases. Further, our data suggest that activated systemic inflammation possibly impacts cancer progression also in the setting of BM. Future trials investigating immune modulating therapies should therefore also consider monitoring systemic inflammation scores and their predictive value to outcome to immunotherapy, eventually finding new predictive markers for a personalised immunotherapy approach in BM patients.

\section{ACKNOWLEDGEMENTS}

We sincerely thank Felix Haupolter for his help in data acquisition. This study was performed within the Ph.D. thesis of Angelika M. Starzer with the title "Immune monitoring in cancer patients" in the N790 doctoral programme at the Medical University of Vienna, Austria. The content of this manuscript was partly presented at the Brain Metastasis Research Symposium in Vienna, Austria, in June 2019 and at the annual conference of the European Society for Medical Oncology (ESMO) in Barcelona, Spain, in September 2019 and nominated as best poster in the CNS session.

\section{AUTHOR CONTRIBUTIONS}

Writing of manuscript draft: A.M.S., A.S.B. Statistical analysis: A.M.S., M.J.M., G.H., A.S.B. Data collection: A.M.S., A.S., A.S., C.D., G.W., B.G., K.D., M.P., A.S.B. Critical revision of important intellectual interdisciplinary content and correction of manuscript: A.M.S., A.S., M.J.M., A.S., C.D., G.W., B.G., K.D., G.H., M.P., A.S.B.

\section{ADDITIONAL INFORMATION}

Ethics approval and consent to participate This study was approved by the ethics committee of the Medical University of Vienna (Vote EK 078/2004). Due to the retrospective design of the study the ethics board waived the informed consent of individual patients. This study was performed in accordance with the Declaration of Helsinki.

Data availability The data supporting the results in this manuscript is saved at a server of the Medical University of Vienna.
Competing interests A.M.S. has received travel support from PharmaMar. A.S.B. has research support from Daiichi Sankyo and Roche, honoraria for lectures, consultation or advisory board participation from Roche, Bristol-Meyers Squibb, Merck, Daiichi Sankyo as well as travel support from Roche, Amgen, Daiichi Sankyo and AbbVie. M.P. has received honoraria for lectures, consultation or advisory board participation from the following for-profit companies: Bayer, Bristol-Myers Squibb, Novartis, Gerson Lehrman Group (GLG), CMC Contrast, GlaxoSmithKline, Mundipharma, Roche, BMJ Journals, MedMedia, Astra Zeneca, AbbVie, Lilly, Medahead, Daiichi Sankyo, Sanofi, Merck Sharp \& Dome, Tocagen. The following for-profit companies have supported clinical trials and contracted research conducted by M.P. with payments made to his institution: Böhringer-Ingelheim, Bristol-Myers Squibb, Roche, Daiichi Sankyo, Merck Sharp \& Dome, Novocure, GlaxoSmithKline, AbbVie. G.H. has received research support from Bristol-Meyers Squibb and Merck. All other authors report no conflicts of interest concerning this specific publication.

Funding information The study costs were supported by a grant from the Margaretha Hehberger-Stiftung assigned to A.S.B. (Project 18085; title: Impact of local and systemic treatments on the inflammatory microenvironment composition in brain metastases). The funding source supported this study by partly financing a Ph.D. student.

Supplementary information is available for this paper at https://doi.org/10.1038/ s41416-020-01254-0.

Note This work is published under the standard license to publish agreement. After 12 months the work will become freely available and the license terms will switch to a Creative Commons Attribution 4.0 International (CC BY 4.0).

Publisher's note Springer Nature remains neutral with regard to jurisdictional claims in published maps and institutional affiliations.

\section{REFERENCES}

1. Achrol, A. S., Rennert, R. C., Anders, C., Soffietti, R., Ahluwalia, M. S., Nayak, L. et al. Brain metastases. Nat. Rev. Dis. Prim. 5, https://doi.org/10.1038/s41572-018-0055y (2019).

2. Moravan, M. J., Fecci, P. E., Anders, C. K., Clarke, J. M., Salama, A. K. S., Adamson, J. D. et al. Current multidisciplinary management of brain metastases. Cancer https://doi.org/10.1002/cncr.32714 (2020).

3. Long, G. V., Atkinson, V., Lo, S., Sandhu, S., Guminski, A. D., Brown, M. P. et al. Combination nivolumab and ipilimumab or nivolumab alone in melanoma brain metastases: a multicentre randomised phase 2 study. Lancet Oncol. 19, 672-681 (2018).

4. Tawbi, H. A., Forsyth, P. A., Algazi, A., Hamid, O., Hodi, F. S., Moschos, S. J. et al. Combined Nivolumab and Ipilimumab in melanoma metastatic to the brain. $N$. Engl. J. Med. 379, 722-730 (2018).

5. Galea, I., Bechmann, I. \& Perry, V. H. What is immune privilege (not)? Trends Immunol. 28, 12-18 (2007).

6. Berghoff, A. S., Fuchs, E., Ricken, G., Mlecnik, B., Bindea, G., Spanberger, T. et al. Density of tumor-infiltrating lymphocytes correlates with extent of brain edema and overall survival time in patients with brain metastases. Oncoimmunology $\mathbf{5}$, e1057388 (2016).

7. Diakos, C. I., Charles, K. A., McMillan, D. C. \& Clarke, S. J. Cancer-related inflammation and treatment effectiveness. Lancet Oncol. 15, e493-e503 (2014).

8. Käsmann, L., Bolm, L., Schild, S. E., Janssen, S. \& Rades, D. Neutrophil-tolymphocyte ratio predicts outcome in limited disease small-cell lung cancer. Lung 195, 217-224 (2017).

9. Fukuda, H., Takagi, T., Kondo, T., Shimizu, S. \& Tanabe, K. Predictive value of inflammation-based prognostic scores in patients with metastatic renal cell carcinoma treated with cytoreductive nephrectomy. Oncotarget 9, 14296-14305 (2018).

10. Riedl, J. M., Posch, F., Moik, F., Bezan, A., Szkandera, J., Smolle, M. A. et al. Inflammatory biomarkers in metastatic colorectal cancer: prognostic and predictive role beyond the first line setting. Oncotarget 8, 96048-96061 (2017).

11. Bumma, N., Jeyakumar, G., Kim, S., Galasso, C., Thakur, M. K., Gadgeel, S. M. et al. Neutrophil lymphocyte ratio (NLR) as a predictive biomarker for PD-1/PD-L1 directed therapy in metastatic non-small cell lung cancer (NSCLC). J. Clin. Oncol. 35, e20633-e20633 (2017).

12. He, X., Li, J.-P., Liu, X.-H., Zhang, J.-P., Zeng, Q.-Y., Chen, H. et al. Prognostic value of $C$-reactive protein/albumin ratio in predicting overall survival of Chinese cervical cancer patients overall survival: comparison among various inflammation based factors. J. Cancer 9, 1877-1884 (2018).

13. Heppt, M. V., Heinzerling, L., Kähler, K. C., Forschner, A., Kirchberger, M. C., Loquai, C. et al. Prognostic factors and outcomes in metastatic uveal melanoma treated 
with programmed cell death-1 or combined PD-1/cytotoxic T-lymphocyte antigen-4 inhibition. Eur. J. Cancer 82, 56-65 (2017).

14. Ishihara, H., Tachibana, H., Takagi, T., Kondo, T., Fukuda, H., Yoshida, K. et al. Predictive impact of peripheral blood markers and C-reactive protein in nivolumab therapy for metastatic renal cell carcinoma. Target Oncol. 14, 453-463 (2019).

15. Shoji, F., Takeoka, H., Kozuma, Y., Toyokawa, G., Yamazaki, K., Ichiki, M. et al. Pretreatment prognostic nutritional index as a novel biomarker in non-small cell lung cancer patients treated with immune checkpoint inhibitors. Lung Cancer 136, 45-51 (2019).

16. Sperduto, P. W., Chao, S. T., Sneed, P. K., Luo, X., Suh, J., Roberge, D. et al. Diagnosis-specific prognostic factors, indexes, and treatment outcomes for patients with newly diagnosed brain metastases: a multi-institutional analysis of 4,259 patients. Int. J. Radiat. Oncol. Biol. Phys. 77, 655-661 (2010).

17. Charlson, M. E., Pompei, P., Ales, K. L. \& MacKenzie, C. R. A new method of classifying prognostic comorbidity in longitudinal studies: Development and validation. J. Chronic Dis. 40, 373-383 (1987).

18. CRAN. Package maxstat. https://cran.r-project.org/web/packages/maxstat/index. html (2020).

19. Chen, Y., Huang, J., He, X., Gao, Y., Mahara, G., Lin, Z. et al. A novel approach to determine two optimal cut-points of a continuous predictor with a U-shaped relationship to hazard ratio in survival data: Simulation and application. BMC Med. Res. Methodol. 19, 96 (2019).

20. Therneau, T. M. Survival analysis $[R$ package survival version 3.1-8]. https://cran.r-project.org/web/packages/survival/index.html (2020).

21. CRAN. Package survminer. https://cran.r-project.org/web/packages/survminer/ index.html (2020)

22. Ohsugi, Y. Recent advances in immunopathophysiology of interleukin-6: an innovative therapeutic drug, tocilizumab (recombinant humanized anti-human interleukin-6 receptor antibody), unveils the mysterious etiology of immunemediated inflammatory diseases. Biol. Pharm. Bull. 30, 2001-2006 (2007).

23. McMillan, D. C. The systemic inflammation-based Glasgow Prognostic Score: a decade of experience in patients with cancer. Cancer Treat. Rev. 39, 534-540 (2013).
24. Klinger, M. H. F. \& Jelkmann, W. Role of blood platelets in infection and inflammation adaptation of thrombopoiesis. J. Inter. Cytokine Res. 22, 913-922 (2002).

25. Palumbo, J. S., Talmage, K. E., Massari, J. V., La Jeunesse, C. M., Flick, M. J., Kombrinck, K. W. et al. Platelets and fibrin(ogen) increase metastatic potential by impeding natural killer cell-mediated elimination of tumor cells. Blood 105, 178-185 (2005).

26. Coussens, L. M. \& Werb, Z. Inflammation and cancer. Nature 420, 860-867 (2002).

27. Chua, W., Charles, K. A., Baracos, V. E. \& Clarke, S. J. Neutrophi//lymphocyte ratio predicts chemotherapy outcomes in patients with advanced colorectal cancer. $\mathrm{Br}$. J. Cancer 104, 1288-1295 (2011).

28. Kao, S. C. H., Pavlakis, N., Harvie, R., Vardy, J. L., Boyer, M. J., Van Zandwijk, N. et al. High blood neutrophil-to-lymphocyte ratio is an indicator of poor prognosis in malignant mesothelioma patients undergoing systemic therapy. Clin. Cancer Res. 16, 5805-5813 (2010).

29. Berghoff, A. S., Wolpert, F., Holland-Letz, T., Koller, R., Widhalm, G., Gatterbauer, B. et al. Combining standard clinical blood values for improving survival prediction in patients with newly diagnosed brain metastases-development and validation of the LabBM score. Neuro Oncol. 19, now290 (2017).

30. Berghoff, A. S. \& Preusser, M. New developments in brain metastases. Ther. Adv. Neurol. Disord. 11, 175628641878550 (2018).

31. Capone, M., Giannarelli, D., Mallardo, D., Madonna, G., Festino, L., Grimaldi, A. M. et al. Baseline neutrophil-to-lymphocyte ratio (NLR) and derived NLR could predict overall survival in patients with advanced melanoma treated with nivolumab. J. Immunother. Cancer 6, 74 (2018).

32. Bilen, M. A., Martini, D. J., Liu, Y., Lewis, C., Collins, H. H., Shabto, J. M. et al. The prognostic and predictive impact of inflammatory biomarkers in patients who have advanced-stage cancer treated with immunotherapy. Cancer 125, 127-134 (2019).

33. Tawbi, H. A.-H., Forsyth, P. A. J., Algazi, A. P., Hamid, O., Hodi, F. S., Moschos, S. J. et al. Efficacy and safety of nivolumab (NIVO) plus ipilimumab (IPI) in patients with melanoma (MEL) metastatic to the brain: results of the phase II study CheckMate 204. J. Clin. Oncol. 35, 9507-9507 (2017).

34. Caponnetto, S., Draghi, A., Borch, T. H., Nuti, M., Cortesi, E., Svane, I. M. et al. Cancer immunotherapy in patients with brain metastases. Cancer Immunol. Immunother. 67, 703-711 (2018). 